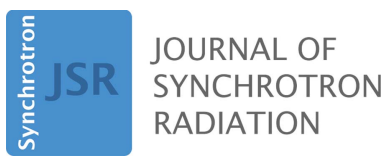

ISSN 1600-5775

Received 12 March 2021

Accepted 28 July 2021

Edited by J. Grünert, European XFEL, Germany

${ }^{1}$ Presented at the PhotonDiag2020 online workshop.

₹ These authors contributed equally to this work.

Keywords: FEL; X-ray spectroscopy; attosecond science; FEL diagnostic.

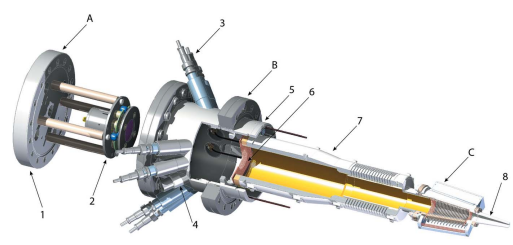

\section{Multi-resolution electron spectrometer array for future free-electron laser experiments ${ }^{1}$}

\author{
Peter Walter, ${ }^{\mathrm{a} *} \neq$ Andrei Kamalov, ${ }^{\mathrm{a}} \neq$ Averell Gatton, ${ }^{\mathrm{a}}$ Taran Driver, ${ }^{\mathrm{b}}$ \\ Dileep Bhogadi, ${ }^{a}$ Jean-Charles Castagna, ${ }^{a}$ Xianchao Cheng, ${ }^{a}$ Hongliang Shi, ${ }^{a}$ \\ Razib Obaid, ${ }^{a}$ James Cryan, ${ }^{\text {a,b }}$ Wolfram Helml, ${ }^{\mathrm{e}}$ Markus Ilchen ${ }^{\mathrm{c}, \mathrm{d}}$ and \\ Ryan N. Coffee ${ }^{a, b}$
}

\footnotetext{
a SLAC National Accelerator Laboratory, 2575 Sand Hill Road, Menlo Park, CA 94025, USA, '⿳⺈ The Stanford PULSE Institute, 2575 Sand Hill Road, Menlo Park, CA 94025, USA, 'European XFEL, Holzkoppel 4, 22869 Schenefeld, Germany, 'Institut fur Physik und CINSaT, Universitat Kassel, Heinrich-Plett-Strasse 40, D-34132 Kassel, Germany, and

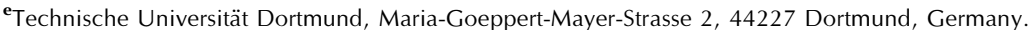

*Correspondence e-mail: pwalter@slac.stanford.edu
}

The design of an angular array of electron time-of-flight (eToF) spectrometers is reported, intended for non-invasive spectral, temporal, and polarization characterization of single shots of high-repetition rate, quasi-continuous, shortwavelength free-electron lasers (FELs) such as the LCLS II at SLAC. This array also enables angle-resolved, high-resolution eToF spectroscopy to address a variety of scientific questions on ultrafast and nonlinear light-matter interactions at FELs. The presented device is specifically designed for the timeresolved atomic, molecular and optical science endstation (TMO) at LCLS II. In its final version, the spectrometer comprises up to 20 eToF spectrometers aligned to collect electrons from the interaction point, which is defined by the intersection of the incoming FEL radiation and a gaseous target. The full composition involves 16 spectrometers forming a circular equiangular array in the plane normal to the X-ray propagation and four spectrometers at $54.7^{\circ}$ angle relative to the principle linear X-ray polarization axis with orientations in the forward and backward direction of the light propagation. The spectrometers are capable of independent and minimally chromatic electrostatic lensing and retardation, in order to enable simultaneous angle-resolved photo- and AugerMeitner electron spectroscopy with high energy resolution. They are designed to ensure an energy resolution of $0.25 \mathrm{eV}$ across an energy window of up to $75 \mathrm{eV}$, which can be individually centered via the adjustable retardation to cover the full range of electron kinetic energies relevant to soft X-ray methods, $0-2 \mathrm{keV}$. The full spectrometer array will enable non-invasive and online spectralpolarimetry measurements, polarization-sensitive attoclock spectroscopy for characterizing the full time-energy structure of SASE or seeded LCLS II pulses, and support emerging trends in molecular-frame spectroscopy measurements.

\section{Introduction}

Electron time-of-flight (eToF) spectroscopy has evolved over the past few decades into a technique capable of providing high-resolution, high collection efficiency, and angle-resolved spectroscopic insights into a broad variety of light-matter interactions in different sample environments at both pulsed and continuous light sources. The instrument presented in this work is optimized for angle-resolved eToF spectroscopy in the gas phase, specifically focusing on those challenges associated with high-repetition-rate X-ray free-electron lasers (XFELs). We report several instrumental advancements to ensure highkinetic-energy resolution with moderate collection efficiency under conditions where tens to hundreds of electrons are 
detected from a single X-ray pulse. From the first dedicated gas-phase eToF (White et al., 1979) to high-resolution and even angle-resolving eToF-spectrometers (Becker et al., 1989; Hemmers et al., 1998; Berrah et al., 1999), advances over the past two decades have led to the first applications of eToF spectroscopy to exploring nonlinear interactions (Braune et al., 2016; Ilchen et al., 2018) and, recently, also time-resolving phenomena at FELs (Hartmann et al., 2018). A complete historic overview is not within the scope of this paper, but the aforementioned milestones are intended to sketch with selected references the direct origins of the presented instrument.

The innovative concept of using an array of more than ten independent time-of-flight spectrometers aligned in the dipole plane of the ionizing field was first pursued by the group of U. Becker, with the goal of capitalizing on the high energy resolution available from eToF spectroscopy while substantially increasing the available angular resolution (Ilchen et al., 2014; Braune et al., 2018). The subsequent developments in the group of J. Viefhaus modified this concept, aiming for higher collection efficiency and the possibility for high retardation potentials which could cover the large photon energy range of 250-3000 eV available at the P04 beamline at the PETRA III synchrotron, DESY, Germany (Viefhaus et al., 2013). This instrument, colloquially named the 'Cookiebox', was successfully employed in experiments at the free-electron lasers FLASH in Germany, FERMI in Italy, and the LCLS in the USA, and inspired the development of a similar device for the European XFEL in Germany (Laksman et al., 2019). Braune $e$ al. presented a conceptually related instrument for spectral and spatial pulse diagnostics at FLASH (Braune et al., 2018). The instrument presented here focuses on the application of non-invasive reconstruction of the exact time, energy, and polarization structure of single X-ray shots for time-resolved experiments at XFELs. It will also allow for the simultaneous acquisition of high-resolution spectra across different kinetic energy regimes, as required for correlationbased photo- and Auger-Meitner electron spectroscopy.

The primary challenges for this new instrument are set by the operation parameters of the upcoming high-repetitionrate LCLS II XFEL. Specifically, LCLS II will feature a $4 \mathrm{GeV}$ continuous-wave superconducting LINear ACcelerator (LINAC) which will produce equally and potentially variably spaced ultrashort X-ray laser pulses at repetitions rates up to $1 \mathrm{MHz}$. Relevant to the spectrometer presented in this work, it will span a photon energy range across the soft X-ray regime (from $0.25 \mathrm{keV}$ to $5 \mathrm{keV}$ ). The superconducting LINAC can deliver X-rays with sub-femtosecond duration via the XLEAP mode of operation (Duris et al., 2020), multi-color pulses (Lutman et al., 2013, 2016a), and (following installation of the afterburner quadrupole DELTA undulators) will allow full polarization control (Lutman et al., 2016b) including timedependent polarization (Sudar et al., 2020). Seeded operation and a variety of pump-probe schemes will further enrich the versatility of LCLS II. Complete characterization of the X-ray pulse properties are critical for the bulk of the science cases of LCLS II (Schoenlein et al., 2015) which target pressing contemporary challenges in ultrafast and nonlinear spectroscopy.

The array of time-of-flight spectrometers presented here, the so-called Multi-Resolution 'Cookiebox' Optimized for the Future of Free-Electron (laser) Experiments - 'MRCOFFEE' - aims to provide an XFEL-optimized single-shot instrument that targets full time-energy reconstruction of XFEL pulses with attosecond temporal resolution as per Hartmann et al. (2018) and (time-dependent) polarization reconstruction as per Lutman et al. (2016b). Targeting attoclock spectroscopy, we have additionally designed for appropriate collection efficiency across multiple degenerate-positioned spectrometers which can be operated with independently controlled retardation fields in order to concurrently measure the timeenergy emission pattern at multiple photoionization and Auger-Meitner electron emission energies. The system is also designed for compatibility at the ultimate $1 \mathrm{MHz}$ repetition rate of LCLS II such that it enables both attosecond scale time-dependent measurements (Driver et al., 2020) and fewfemtosecond scale nonlinear experiments (Saalmann \& Rost, 2020), expected to play guiding roles in the next decade of FEL science.

A principal strength of the spectrometer array lies in the high time resolution of the micro-channel-plate (MCP) based detectors. The MCP detectors record electron counts from single pulses at high repetition rates with excellent time resolution and narrow signal-pulse envelopes (450 ps FWHM). This narrow pulse width affords the time resolution necessary to realize the required energy resolution, even for the very short flight times associated with the upper end of a variable pass-energy window. The MCP detectors can also be run in analog mode, whereby the current through the MCPs is measured. This analog mode, as opposed to counting mode, can be used to accommodate conditions where single-electron counting is either prohibitively difficult or not desirable, both of which are frequently the case at XFELs.

\section{Hardware}

As discussed above, the MRCOFFEE instrument for diagnostic and experimental measurements will record electron spectra over independently defined energy ranges with high resolution for ultra-intense XFEL pulses. This section describes the engineering challenges required for this instrument and is organized by different general components: the main chamber, the eToF spectrometers, and the target delivery and X-ray alignment tool.

\subsection{The main chamber}

The main chamber is engineered to house 16 eToF spectrometers orientated azimuthally about the axis of X-ray propagation and equally spaced by $22.5^{\circ}$. There are an additional four spectrometers oriented at the so-called 'magic' angle of $\theta=54.7^{\circ}$ relative to the horizontal X-ray polarization outside of the dipole plane by $\varphi=42.87^{\circ}$. At this particular angle the emitted electron signal is independent of angular 
anisotropies relative to the X-ray propagation axis and therefore directly proportional to total yield (Kivimäki et al., 1998). Measurements at the magic angle also aid the retrieval of laboratory frame angular asymmetries in electron emission patterns (Patanen et al., 2013). Two of these additional spectrometers are oriented in the forward direction of the light propagation direction and two in the backward direction, in order to enable investigations of non-dipole effects. The chamber has additional ports for four non-magnetic bearing turbomolecular vacuum pumps, sample delivery, X-ray beam diagnostics, vacuum feedthroughs, and viewports.

A centering ring mounted at the center of the chamber and common

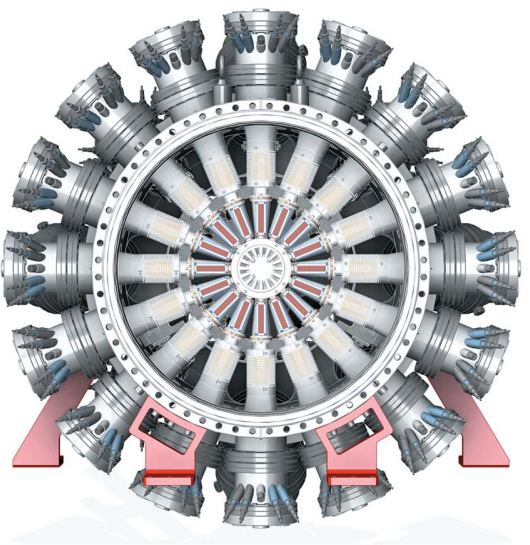

(a)

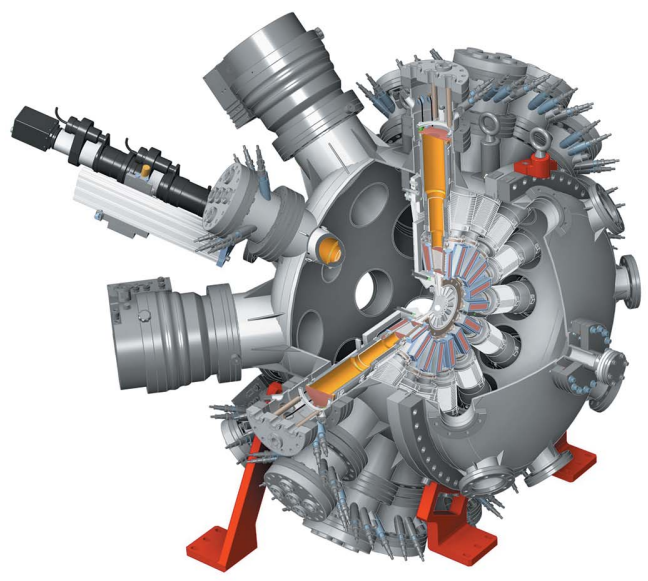

(b)

Figure 1

(a) Axial view of the detector array along the direction of X-ray propagation, showing the 16 azimuthal spectrometers. (b) Section view partly showing 'magic'-angle spectrometers, turbomolecular pumps, and an interaction-viewing camera. to all spectrometers allows for precise mechanical alignment of all spectrometers to a common interaction region with better than $100 \mu \mathrm{m}$-diameter intersection volume. This precise mechanical alignment, similar to previous designs, relieves the need for independent manipulators for each spectrometer.

Any electrons encountering non-homogeneous magnetic fields in flight to the MCP detectors experience a distortion in measured yield and resolution. This effect is particularly severe for low-kinetic-energy electrons. To avoid any undesired effects of this nature, both the main chamber and the spool pieces on which the spectrometers are mounted (see Section 2.2) are manufactured of high-magnetic permeability mu-metal. Along with mu-metal bridging rings which facilitate guiding magnetic field lines across the stainless steel flanges, the mu-metal construction of the chamber and spools significantly reduces the magnetic field in the flight path of the electrons through the spectrometers (see Figure 2).

Magnetic materials such as nickel and stainless steel were avoided for internal components whenever possible to further limit the deleterious effect of stray magnetic fields. The main chamber schematic is shown in Figure 1 and the simulated magnetic shielding is shown in Figure 2 for magnetic fields (B) vertically perpendicular to the X-ray trajectory (top row) and horizontally perpendicular to the X-ray trajectory (bottom row).

\subsection{The spectrometers}

The eToF spectrometers require a reliable design that can provide high resolution and collection efficiency at any userspecified energy region. A uniform modular design ensures serviceability and allows reconfiguration or replacement for selective angular configurations. All spectrometers operate simultaneously to measure angle-resolved electron spectra, addressing the target applications of attosecond angular streaking measurements, LCLS II diagnostics, and a broad range of scientific measurements.
Our spectrometer design is presented in detail in Figure 3 with dimensions and electron-acceptance diameter summarized in Table 1. Electrons entering the eToFs first encounter a series of electrostatic lenses which retard and partially collimate the electrons to improve energy resolution. Electrons then traverse a gradient-free drift tube and pass through a meshed aperture at the exit of the drift region. Immediately upon exiting the flight tube, electrons are pre-accelerated into a commercially available MCP detector [Hamamatsu model F9890-31(-32)]. The spectrometer assembly is mounted in a mu-metal spool piece which directly supports the lensing section and the drift tube assembly. The MCP detector is a separate component which is re-entrant to the mu-metal
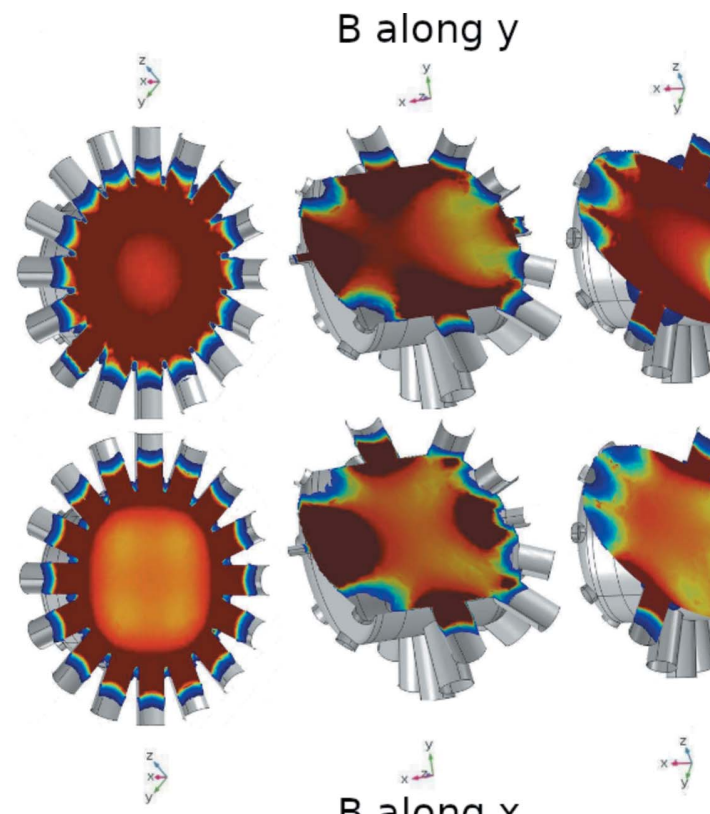

Figure 2
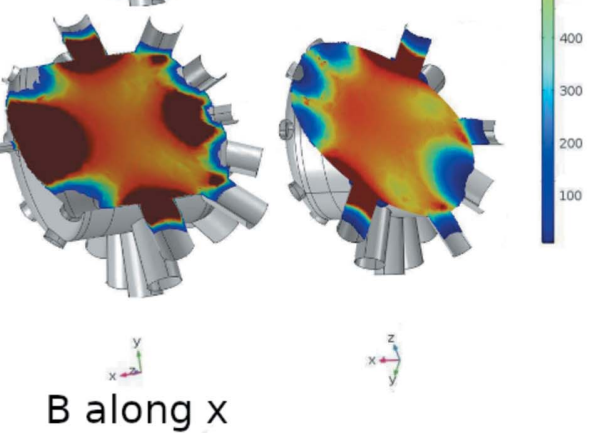

Magnetic shielding factor (attenuation factor) calculations for the final experiment chamber design. Magnetic attenuation calculation performed by Scientific Magnetics (http://www.scientificmagnetics.co.uk/). 
Table 1

The four principal regions of the spectrometer, defined by the distance from the X-ray/target interaction point.

The relative solid angle acceptance is noted in \% of a unit sphere.

\begin{tabular}{|c|c|c|c|c|}
\hline Region & $\begin{array}{l}\text { Start } \\
(\mathrm{mm})\end{array}$ & $\begin{array}{l}\text { Stop } \\
(\mathrm{mm})\end{array}$ & $\begin{array}{l}\text { Stop aperture } \\
\text { diameter }(\mathrm{mm})\end{array}$ & $\begin{array}{l}\text { Relative solid } \\
\text { angle (\%) }\end{array}$ \\
\hline Interaction & 0.0 & 25.0 & 4.36 & 0.189 \\
\hline Nose & 25.0 & 80.0 & 15 & 0.180 \\
\hline Lens/retardation & 80 & 132.8 & 25 & $0.220 \dagger$ \\
\hline Drift & 125 & 396.3 & Up to $57 \neq$ & Up to 0.129 \\
\hline
\end{tabular}

$\dagger$ Slightly larger opening of $0.220 \%$ than upstream acceptance limitation. $\$$ Current MCP diameter is $27 \mathrm{~mm}$ giving $0.029 \%$ angular acceptance

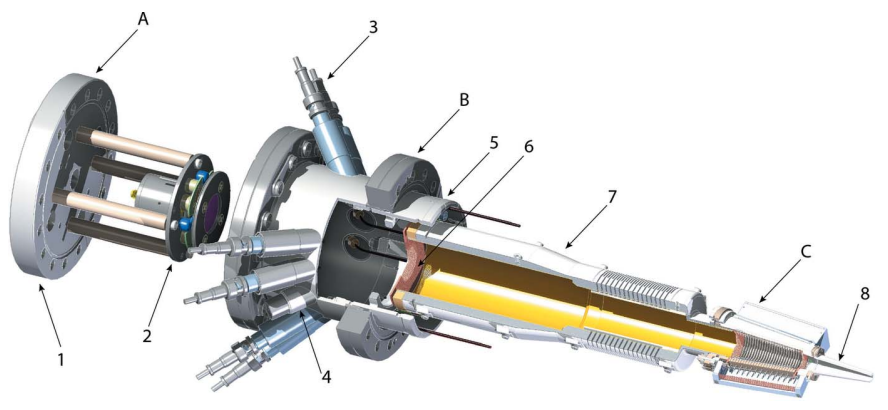

Figure 3

The spectrometer. Shown are (A) the MCP assembly, (B) the flight-tube assembly, and (C) the lens-stack assembly (see Figure 4 for detailed view). The MCP assembly consists of a (1) feedthrough flange with five miniflange openings for individual feedthroughs and (2) the fast-response MCP-detector. The drift-tube assembly consists of (3) eight high-voltage feedthroughs, (4) two nine-pin high-voltage multi-pin feedthroughs, (5) a mu-metal bridge, (6) the end-of-drift-tube mesh, and (7) the drift tube. (8) shows the flight-tube nose cone.

spool, in order that it benefits from the magnetic shielding, but remains separately mounted for ease of replacement, reconfiguration and service. The mu-metal spool piece is equipped with ten high-voltage (HV) feedthroughs; eight which are compatible with $6 \mathrm{kV}$ low-current supply and two nine-pin feedthroughs compatible with up to $1 \mathrm{kV}$. The total of $26 \mathrm{HV}$ feedthroughs allows for future development of more finely controllable electrostatic potentials. The drift tubes incorporate a flexible section in order to accommodate thermal expansion during vacuum baking and straightforwardly support the mechanical register of the spectrometer-nose section into the socket of the centering ring.

\subsection{The compound lens}

The electron collection of the individual spectrometers is controlled by a series of 25 copper blades positioned at the front of the spectrometer modules, as shown in Figure 4. These blades form a compound electrostatic lens to provide energy retardation and partial recollimation. Fine copper meshes with transmission greater than $90 \%$ are positioned at the front and back of the compound lens. The meshes ensure that the electrostatic potential is flat outside of the lens stack, enabling free propagation to the $\mathrm{MCP}$ detector. The electric potential steadily drops (becomes increasingly negative) along the blade

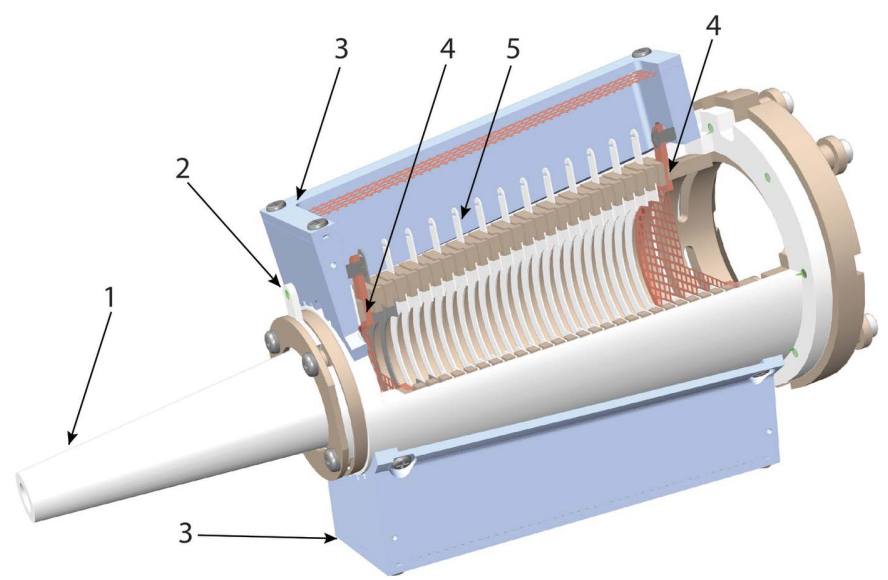

Figure 4

Cross-section of the electrostatically retarding compound lens. Shown are (1) the nose cone and respective (2) HV connection, (3) RF shielding cages for the resister bridge, (4) copper meshes at the beginning and end of the retardation lenses and (5) the stack of 25 lensing blades.

stack in the direction of the electron propagation, continually lowering the kinetic energy of electrons to increase the respective energy resolution. The blades and fine copper meshes are electrically connected via a resistor bridge which, combined with voltages supplied to the endpoints of the resistor bridge and optional voltage supply points along the blade series, determine the field gradient of the compound lens.

The selection of the resistor bridge's values and voltagesupply attachment points was driven by the goal of creating an electric lens with $0.25 \mathrm{eV}$ resolution within a chosen $10-75 \mathrm{eV}$ energy window for retarded electrons (see explanations below). We first investigated a $1 / r$ potential profile with resistor-bridge node values shown in Figure 5(a), where the nodes correspond to the front mesh at node 0 , blades from nodes 1 to 25, and the back-mesh at node 26. This configuration preserves high energy resolution but sacrificed collection efficiency, particularly at high retardation. In this case, we define the total retardation potential by setting both the mesh- and drift-region potential to $V_{\text {ret }}$, while holding the front mesh and nose to ground potential.

In addition to a low collection efficiency, this configuration would bend the outermost-accepted electron trajectories back towards the center of the MCP, crossing over the center line in the drift region (not shown). This effect will lead to a region of high-density electron hits on the MCP detector, which may saturate the detector and as a result decrease the collection efficiency of the spectrometer for particular pass energies. This is the well known chromatic 'sausage' effect, whereby some energies are over-focused, others are focused into the center of the detector, and yet higher energies are under-focused. This effect is of particular concern for the use with the highrepetition-rate LCLS II since the cumulative effect of a high number of electrons being delivered to a small spot over an extended period of time could be potentially damaging for an MCP. We therefore ensure that no energies are tightly focused into the center of the MCP. 


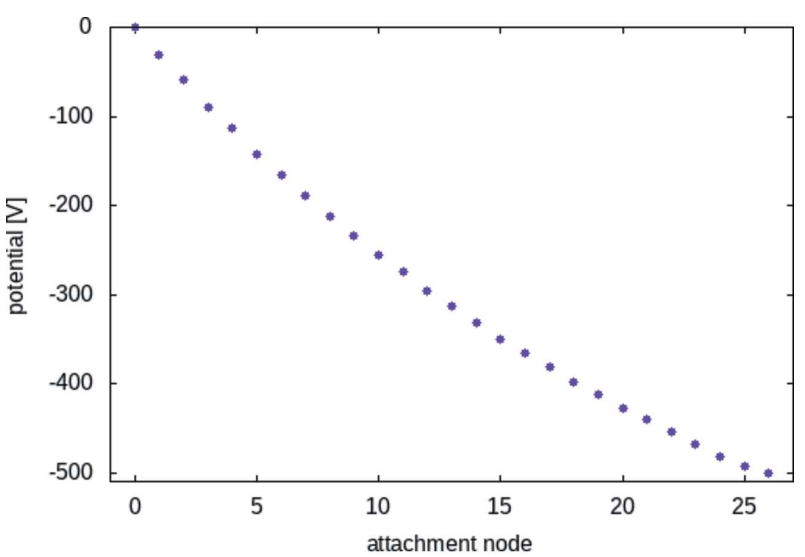

(a)

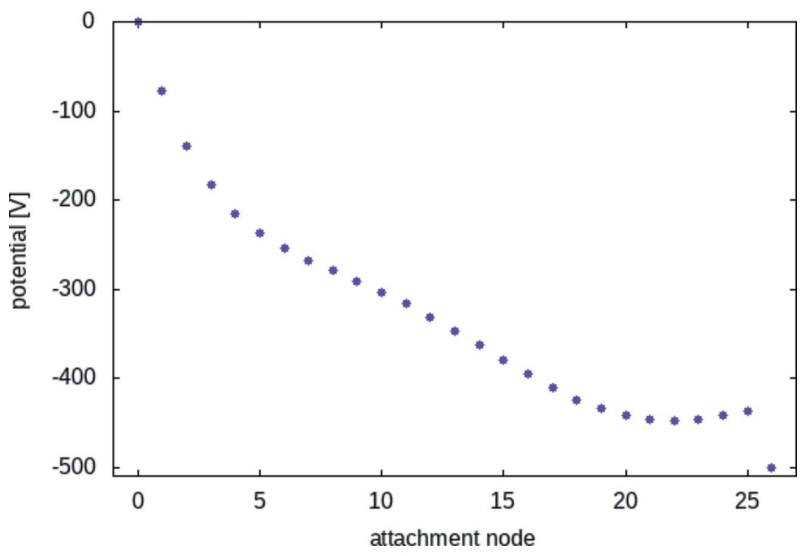

(b)

Figure 5

Potentials along the nodes of the (a) $1 / r$ compound-lens design and $(b)$ Nelder-Mead optimized design. Node 0 corresponds to copper mesh at the entry of the compound lens, nodes 1-25 correspond to the 25 blades, and node 26 corresponds to the copper mesh at the end of the compound lens. The voltages plotted here are for a starting potential of $0 \mathrm{~V}$ and a final potential of $-500 \mathrm{~V}$, or $500 \mathrm{~V}$ of electron retardation. The voltage profile scales linearly with desired retardation value.

A proposed alternative to the $1 / r$ potential configuration is presented in Figure 5(a). The alternative was found using the Nelder-Mead optimization algorithm (Nelder \& Mead, 1965) with an objective function which optimizes a monotonic mapping of the acceptance solid angle to the $27 \mathrm{~mm}$-diameter MCP detector area, avoiding the chromatic 'sausage' effect. The optimization used SIMION simulations to provide feedback of compound lens performance and trajectory crossings at every iterative step. The optimized compound lens has the desired energy resolution for retarded electrons while maintaining high collection efficiency, as will be shown in subsequent Section 3. The final part of this compound-lens configuration employs a sudden potential step to collimate the electron trajectories onto the MCP detector, as seen between nodes 25 and 26 in Figure 5(b).

\subsection{Gas delivery and interaction alignment}

The main sample delivery apparatus is an effusive gas nozzle pressurized via a flow controller. The sample delivery rod can be set to high voltage in the event of charging, and also

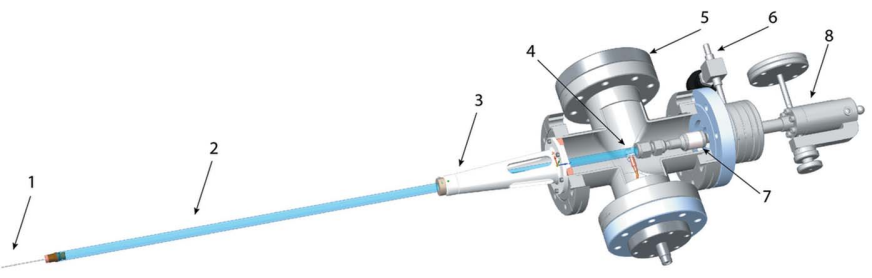

(a)

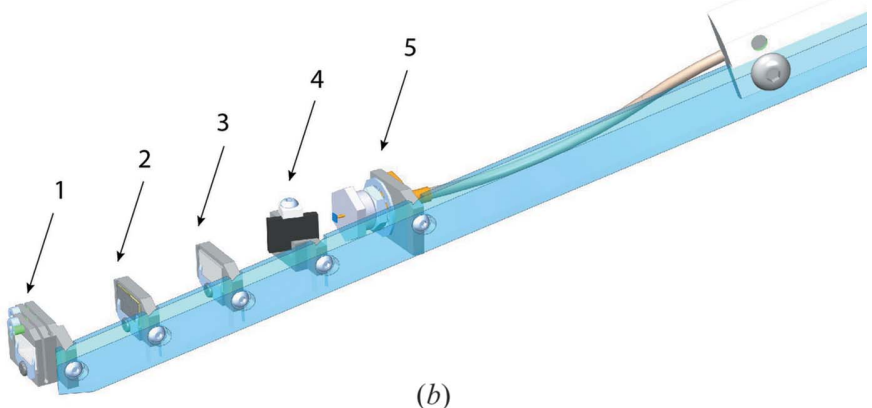

(b)

Figure 6

(a) Gas-sample injection assembly. Shown are the (1) sample-gas nozzle,

(2) the gas-support tube, (3) the alignment stabilizer, (4) HV connection,

(5) additional feedthrough flange, (6) pump-and-purge port, (7) electric isolating inlet coupling, and (8) the leak valve. (b) Interaction alignment and X-ray focus diagnostic paddle. Depicted are (1) the flexure holder for the pinhole array, (2) and (3) the YAG and filter holders, (4) the opticallaser scatter target and (5) the timing diode.

enables the deliberate application of an electrostatic field. The in-vacuum interaction end of this rod is insulated from the end of the gas-inlet source, with voltages applied via a HV feedthrough at the source cross. Two sub-D feedthroughs can be used to install a sample heating line up to the tip (see Figure 6). For precise alignment, the sample delivery apparatus is mounted on a motorized three-way manipulator.

The instrument is equipped with a multi-purpose diagnostic paddle reaching into the interaction region, mounted on a three-way manipulator, to accommodate X-ray focus diagnostics and beam positioning as well as coarse X-ray arrivaltime determination. For commissioning, calibration, and alignment of the TMO wavefront sensor, the first position of the diagnostic paddle (1) is a three-axis flexure that allows alignment of a pinhole array wafer perpendicular to the X-ray propagation vector. Positions (2) and (3) host $10 \times 5 \mathrm{~mm}$ wafers such as YAG, imprint samples, and/or filters that can be individually selected depending on the experimental requirements. Position (4) is a fluorescence target used with a highspeed photodiode (5) (Hamamatsu, currently discontinued) for coarse X-ray timing; paddle motion allows for both options: fluorescence or hitting the diode directly. The sample paddle is mounted from the back of the chamber with $\theta=69.3^{\circ}$ relative to the horizontal $\mathrm{X}$-ray polarization outside of the dipole plane by $\varphi=-22.2^{\circ}$.

\section{Preliminary results}

A principal challenge for MCP-based instruments such as the one presented here is the consideration of the tolerable number of electrons per shot which, for high-repetition-rate 
XFELs, can affect the single-shot capability as well as longevity of the MCP. The prospected leap of orders of magnitude in peak and average brightness of LCLS II creates unprecedented challenges in this regard.

The maximum tolerable number of electrons detected by a single detector from one single-shot is largely set by the charge-replenishment rate of the MCPs between each pulse, while longevity is a function of the MCP-aging characteristics under continuous high signal conditions.

On the one hand, we prefer high signals for the sake of enabling single-shot spectroscopy and diagnostics, but, on the other hand, the analog operation mode will most likely be incompatible with an effective $\mathrm{MHz}$ repetition rate. For this reason, we plan to enable both modes selectively for the requirements of individual experiments. In counting mode, we will, in a first step, run the MCP bias voltage to the lowest end of the linear-gain regime, compensating the potentially small counting-mode signals with broad-band signal amplification (discussed below), immediately at the output of the MCP detector flange. To enable the counting mode on a more robust voltage for the linear amplification scheme of the MCPs will, however, require demanding levels of ultrahigh vacuum, i.e. a dilute gas target and/or relatively low irradiation levels.

The challenge of forming statistically robust single-shot spectra, as, for example, acquired in angular streaking during previous experiments (Hartmann et al., 2018) and used for XFEL-pulse-resolved diagnostics (Laksman et al., 2019), will demand balancing the chamber-vacuum base pressure, sample-gas flow and X-ray $I_{0}$ variation. In particular, this operation must rely on a well understood linear amplification by the MCPs in order to be able to obtain valid ratios of numbers of electrons at individual kinetic energies and yields.

An important limitation in this regard concerns avoiding space charge-induced degradation of the energy resolution due to Coulombic interactions in the interaction volume. We base our following estimates for this limit on previous experiences with the original Cookiebox of J. Viefhaus, and its equivalent unit at the European XFEL (Hartmann et al., 2016, 2018; Lutman et al., 2016b). The preliminary performance of a spectrometer with the optimized compound lens is characterized using the SIMION simulation software. Figure 7 shows simulated ToFs at various retardation settings for Gaussian energy peaks with $\sigma=0.0625 \mathrm{eV}$, separated by $0.25 \mathrm{eV}$. The simulation is performed for electrons that are born with high kinetic energy and are then retarded by the compound lens to have final kinetic energies near $100 \mathrm{eV}$. The individual ToF traces are references such that the earliest, or most energetic, peak of $100 \mathrm{eV}$ post-retardation arrives shortly after $t=0$, in order to compare the ToF resolution for electrons retarded by different values. Even at these energies, ideal ToF histograms binned by the digitizer sampling period of $0.166 \mathrm{~ns}$ are structured, although sub-optimal. We supplement our compound lens design with digital signal processing as summarized in Section 4 to further resolve hits and attain sub-sampling resolution.

Figure 8 shows the fractional angular collection as a percentage of unit spherical emission. We restrict the pass

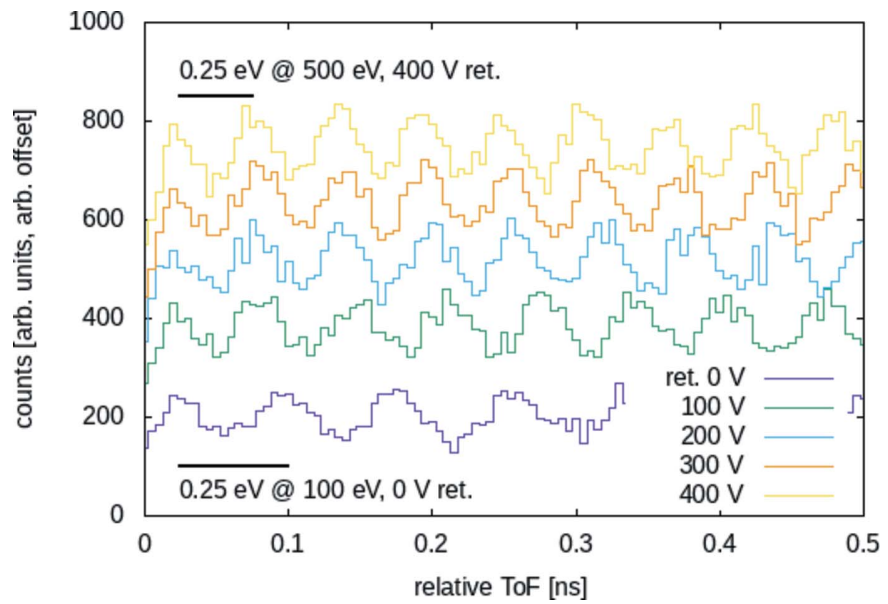

Figure 7

Simulated comparison of the ToF resolution versus retardation for a spectrometer with the optimized compound lens configuration. The individual traces are referenced such that $t=0$ is shortly prior to the arrival of electrons with post-retardation energy of $100 \mathrm{eV}$. These are followed by a series of subsequently lower energy (later arrival) peaks with Gaussian distributions of $\sigma=0.0625 \mathrm{eV}$ separated by $0.25 \mathrm{eV}$.

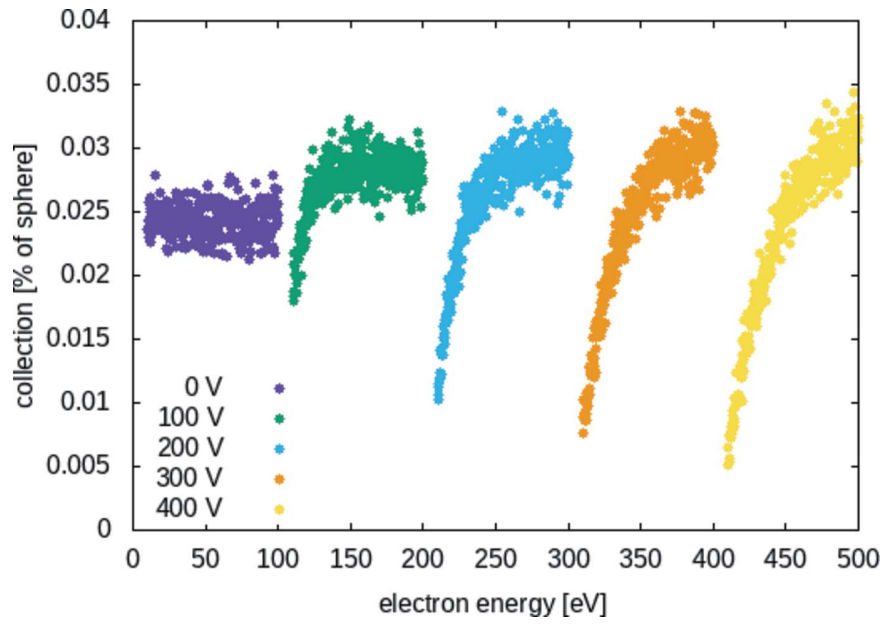

Figure 8

Simulated collection efficiency comparison for a single spectrometer at various electron energy ranges with corresponding retardation potentials. Retardation is noted in the key and simulation results are shown for 10$100 \mathrm{eV}$ above each retardation potential.

energy window to above $10 \mathrm{eV}$, due to a potential preponderance of secondary electron emission below $10 \mathrm{eV}$ that would otherwise obscure the desired target molecule spectra. This simulation shows that the optimized field gradient retards high-energy electrons without significantly sacrificing collection. The absolute efficiency is set by the solid-angle size of an individual spectrometer's entry aperture and corresponds to a maximum possible collection percentage of $0.189 \%$ as noted in Table 1.

\section{Signal processing}

The full instrument allows for up to 20-fold parallel signal readouts that can in turn be used for real-time data sorting, veto, and statistical analysis of downstream detectors. At its 
most simple form of veto, low latency results will allow users to choose on the fly only those X-ray shots with desirable pulse parameters; at its most advanced, semi-autonomous control could automatically drive the XFEL toward conditions that are likely to expose more information-rich regions of parameter space. For example, in attosecond physics experiments (Hartmann et al., 2018; Driver et al., 2020), one could ultimately wrap a control loop around pump-probe delay settings in the XFEL such that the experiment would self-tune to information-rich delay and multi-pulse energy settings. In polarization-based studies, one could use the time- and spectrum-resolved polarization results to aggregate desired shots or even control the non-trivially shaped polarization states (Sudar et al., 2020) to drive toward conditions that expose chiral dynamics. At the very least, the instrument would serve as the first data reduction step for LCLS II and LCLS-HE experiments, providing a critical function for experiments that will soon incorporate $2 \mathrm{D}$ area detectors capable of serving the $1 \mathrm{MFps}$ imaging rate. We have co-designed the detector array along with analysis algorithms that will accommodate lowlatency decisions based on X-ray pulse characteristics. We will implement these algorithms in a data-flow paradigm using FPGA-based streaming analysis that divides X-ray pulse reconstruction into stages of increasing complexity. Although the deeper layers of analysis, performed later in the data transformation chain, are beyond the scope of this instrumentation manuscript, we note that they presuppose a socalled 'featurized' representation of the single-shot electronenergy spectrum. It has been recently shown that an FPGAimplemented inference engine for coarse-grained X-ray properties require the energy-domain histogram rather than the direct time-of-flight spectrum (Therrien et al., 2019). We plan to produce the energy domain histogram directly in the FPGA on the waveform digitizers themselves and so have chosen digitizers that allow for custom firmware (Abaco Systems Inc.).

Given the hit rate and space-charge limits, we will adjust the target gas density to limit the incidence hit rate to below 100 counts per shot per MCP detector. We therefore focus on the counting regime for the MCP detector signals, leveraging the fast rising edge of the electron-cascade signal for attaining sub-sampling flight time resolution. We also reduce the total charge needed to register a hit and favor fast fall time as well since the individual spectra will likely be quite congested in this rather high-count-rate regime. For initial demonstrations, we have chosen the Hamamatsu F9890-31(-32) detector assembly which supports a FWHM rise-time resolution below $0.5 \mathrm{~ns}$. In a first step, we rely on external high-bandwidth signal amplification to accommodate reduced MCP gain in order to mitigate charge depletion while preserving sensitivity and rising-edge bandwidth.

In order to achieve the best resolution in the energy representation, we recognize that the precision in timing information is contained in the high-frequency region of the signal power spectrum. Figure 9 follows our signal processing algorithm whereby we read the amplified signal as discussed in Section 3. The minor ticks in Figure 9 represent the expected digitization step size for the TMO instrument. For the sake of testing, however, waveforms were measured with a $40 \mathrm{GS} \mathrm{s}^{-1}$ (20 GHz bandwidth) LeCroy digital oscilloscope. We optimized the bias voltage across the MCP in order to optimize the signal-to-noise ratio for frequencies above $1 \mathrm{GHz}$.

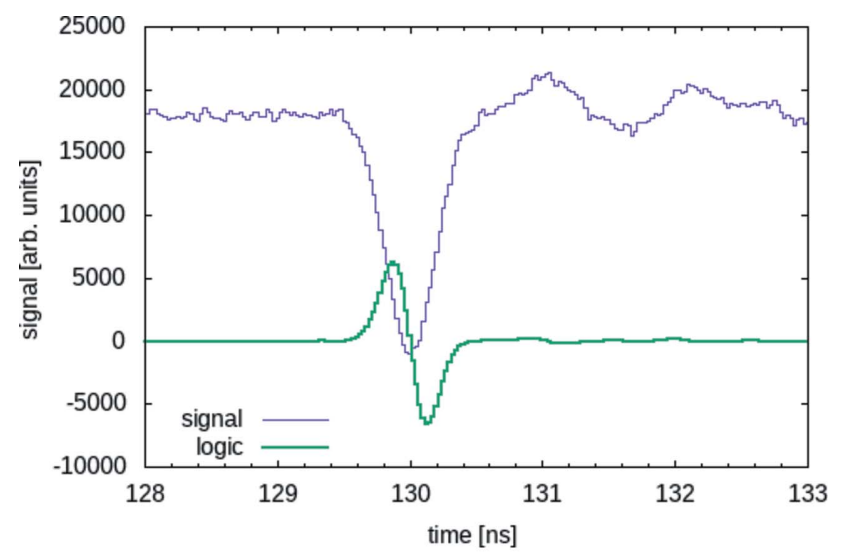

(a)

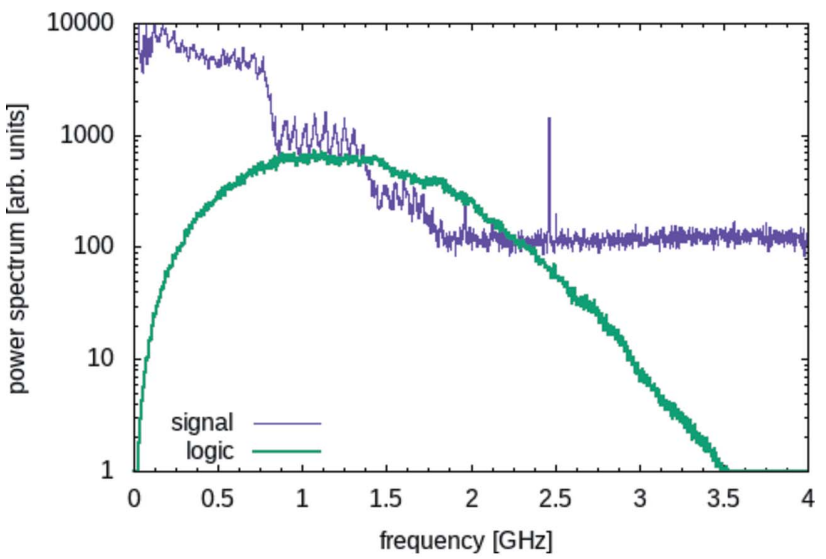

(b)

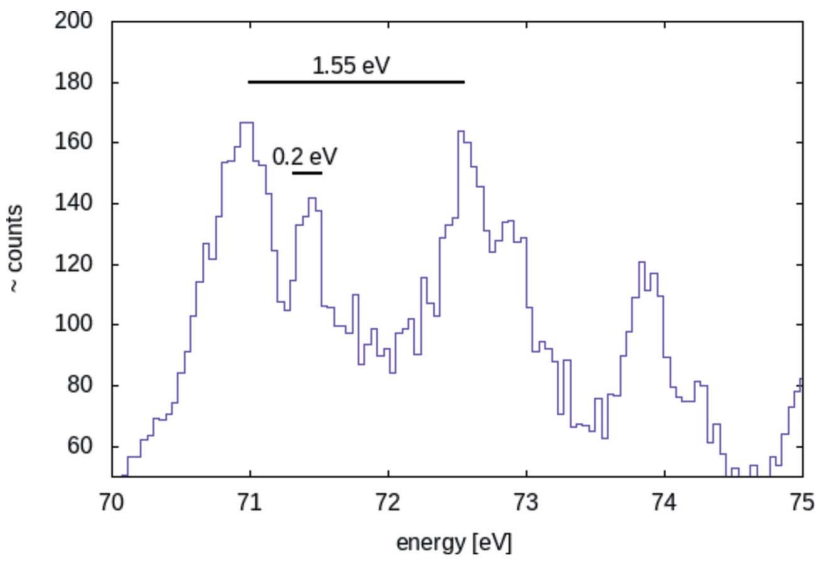

(c)

Figure 9

(a) Waveform with an example signal amplifier minicircuits model CMA$83 \mathrm{LN}+$ and the 'logic' waveform as described in the text. (b) The average power spectrum of the signal and logic waveforms. The logic waveform preserves the high frequency region of the signal but suppresses the low frequency and as well mitigates the residual post-pulse overshoot. (c) Measured argon ATI spectrum with $70 \mathrm{~V}$ of accelerating potential applied. 


\section{Diagnostic and scientific application}

The experimental paradigm of 'measure-and-sort' has grown ubiquitous to the XFEL community. Be it related to the X-ray arrival-time diagnostics (Bionta et al., 2014), the numerous electron bunch diagnostics, or the spectral properties of the X-ray pulses themselves (Driver et al., 2020), users have become expectant of and profited significantly from singleshot analysis for sorting and other algorithmic treatment of the science data accordingly for each unique XFEL shot.

The instrument presented here is expected to serve this paradigm by providing single-shot reconstruction of the full multi-color pulse intensities, photon energies, and polarization states in the wide spectral range, as has been recently enabled by the LCLS II variable-gap undulators. Of central importance is the use of time-of-flight to encode electron energy; this relaxes our need to extract photo- and Auger-Meitner electron spectra from high-resolution imaging sensors. With this technique, we use the representation of energy as a drift time within the $\mu$ s window such that the signals are fully compatible with the highest repetition rates of the LCLS II.

\subsection{Diagnostics}

Since our instrument aims at a simultaneous multi-edge characterization of photo- and Auger-Meitner electron spectra with high energy resolution, it will enable spectral diagnostics in an extended but also redundant, i.e. enhanced collection efficiency, scheme. This can be particularly powerful in multi-color experiments with large color separations and/or in need of optimal conditions for each of the colors under consideration. Not only will it non-invasively complement high-resolution grating-based spectrometer measurements for cumulative characterization of averaged pulses, it will also enable cross referencing of multiple spectrometers with ideally equivalent information, thereby efficiently neglecting systematic effects of trajectory alterations that could compromise the diagnostic validity. The design is developed in a way that the instrument is anticipated to also operate in the hard X-ray regime, depending on the photon energies and the targeted absorption edges of available gas targets, enhancing the interdisciplinary use of this instrument within the facility's aims and scientific vision. The past years have seen the extension of the novel and powerful approach of attosecond angular streaking (Eckle et al., 2008) to the time-energy characterization of XFEL pulses (Hartmann et al., 2018; Heider et al., 2019). The experimental results provide direct information about the full time-energy distribution of individual stochastic SASE X-ray pulses with attosecond resolution, revealing X-ray pulse and sub-spike duration, intensity substructure, and chirp. Angular streaking uses a circularly polarized optical laser to dress target atoms or molecules such that photoionization incurs a rotating energy sweep as a sort of optical carrier clock (Constant et al., 1997; Eckle et al., 2008). Thus, the temporal intensity distribution of the XFEL pulse is mapped to the energy distribution of the streaked photoelectron spectra with the angular distribution providing the necessary temporal characterization of FEL pulses with attosecond resolution (Kienberger et al., 2002; Helml et al., 2014; Kazansky et al., 2016; Li et al., 2018). In combination with a long-wavelength laser field, our instrument will measure directly the X-ray profile (Hartmann et al., 2018) via angular streaking, making available X-ray pulse duration measurements where the use of the $\mathrm{x}$-band transverse cavity (XTCAV) is precluded by the high repetition rate of the LCLS II. This use applies even at low repetition rate since short X-ray pulses suffer from comparable electron/X-ray slippage such that XTCAV interpretation no longer properly represents bunch dependent lasing. This need for the angular-streaking alternative of XTCAV will become an increasing concern as the community explores multi-stage amplification schemes (Lutman et al., 2016b; Amann, 2018; Emma et al., 2017). The increasing interest in polarization control further motivates the use of MRCOFFEE as a time-resolving X-ray pulse polarimeter. Given the development of time-dependent polarization states of XFEL pulses as in Sudar et al. (2020), online measurement of the polarization state as in Lutman et al. (2016b) combined with the attosecond resolution of Hartmann et al. (2018) and Heider et al. (2019) portends active use as a spectral and polarization diagnostic during tune-up, demonstration, and user use of such novel operation modes. At the LCLS II, the possibility of effectively using a reverse taper scheme to deliver circular polarization and of seeding the Delta undulator with the Fresh-slice technique (Lutman et $a l ., 2016 b$ ) will require online measurement of the polarization characteristics to optimize the cross-polarized undulator beams. Beyond the use for setup of such novel FEL operation modes, this diagnostic capability will allow on-the-fly sorting based on the polarization state of individual shots, bringing also time-dependent polarization into the 'measure-and-sort' paradigm. On the nanosecond front, our instrument can be used to identify nanosecond-separated pulse intensities when running so-called 'multi-bucket' modes. Using atomic or molecular photo- and Auger-Meitner electron spectra with sharp spectral features will allow the identification of timeshifted copies of the spectra, thus revealing the separation and even intensity variation for the multi-bucket pulse train with sub-ns resolution.

\subsection{Science}

Angle-resolved photo- and Auger-Meitner electron spectroscopy (ARPES and ARAES, respectively) enable XFELs to tackle a variety of desired scientific objectives across different sample environments and scientific disciplines. Building on our history of XFEL-based molecular spectroscopy (Cryan et al., 2010, 2012), one of the driving goals for this instrument is enabling advanced soft-X-ray spectroscopic methods. Targeting simultaneous molecular photoelectron and resonant Auger-Meitner studies, we have designed for $0.25 \mathrm{eV}$ resolution on both incoming photon energy reconstruction and outgoing electron kinetic energy resolution. The angle-resolving spectroscopic capabilities alone of this instrument will serve the broader molecular spectroscopy community (Hoshino et al., 2006; Simon et al., 2014; Goldsztejn 
et al., 2016) as well as the emerging attosecond-resolved studies enabled by angular streaking. The scope of the associated methodological developments extend well beyond the familiar investigations of the emission characteristics of, for example, crystal lattices, simple atomic or molecular systems, and their orbital structure and dynamics. Not only do angleresolved electron spectra from a dilute gas target non-invasively reveal the state and degree of circular polarization (Lutman et al., 2016b) and the attosecond time-energy structure of XFEL pulses (Hartmann et al., 2018; Li et al., 2018; Heider et al., 2019), but also it simultaneously grants access to the electron structures and dynamics in small quantum systems. Depending on the experimental scheme, our instrument can be employed together in situ or serially in parallel with experiment specific instruments along the beamline. The experimental possibilities thus opened up could be applied to the investigation of general light-matter interaction of atomic and molecular targets, ultrafast reaction pathways for photolysis processes in prototypical and eventually complex organic molecules, the study of fundamental charge dynamics, for example, in water to deduce information about DNA damage. Excitingly, these research opportunities quite generally pave the way to ultrafast X-ray pump-probe experiments at the attosecond frontier (Li et al., 2021; Saalmann \& Rost, 2020), setting the stage for nonlinear attosecond $\mathrm{X}$-ray science that can resolve electron dynamics at their natural time scales (Haynes et al., 2021). Inspired by the broad applicability of MRCOFFEE to both scientific and diagnostic tasks, we here present a few selected research avenues for fundamental science applications. We will specifically spotlight three gas-phase applications that will be enabled, advanced, or leveraged by the XFEL-tailored design of the instrument, namely attosecond X-ray physics, polarization control, and molecular-frame spectroscopy. The novel technique of angular streaking can non-invasively retrieve every shot's time-energy structure and duration with attosecond resolution. As introduced above, it was demonstrated that even X-ray pump/ $\mathrm{X}$-ray probe experiments with sub-fs pulses are feasible by sorting for appropriate delays between SASE spikes. A much more advanced control and delay-resolution has meanwhile been enabled (Li et al., 2021), bringing time-resolved XFEL $\mathrm{X}$-ray science fully into the attosecond regime (Hartmann et $a l .$, 2018). With the improved design of MRCOFFEE, we will be able to follow up on the recent results of attosecond angular streaking and carry them further. In line with the recent demonstration of XLEAP at the LCLS (Duris et al., 2020) together with emerging X-ray polarization methods (Sudar et al., 2020), this instrument will fully support the development of new operation modes with high-energy attosecond pulses and innovative measurement schemes. A potentially critical concept is that of attoclock ptychography (Schweizer et al., 2018) in which the application of timedomain ptychography to angular photoelectron streaking is proposed. Originally, space-domain ptychography was used to solve the phase problem in crystallography (Hoppe, 1969) by iteratively reconstructing an image from a series of overlapping far-field diffraction images. Attoclock ptychography operates similarly but in the time-frequency domain whereby angular streaking spectra replace the diffraction images of conventional ptychography. Upon close inspection of Schweizer et al. (2018), we find that a compromise in energy resolution of about $0.25 \%$, e.g. $0.25 \mathrm{eV}$ in the $10-75 \mathrm{eV}$ window, achieves an acceptable reconstruction accuracy that only improves with diminishing returns until the spectrometer resolution becomes an order of magnitude better than achievable for anything other than an angle-integrating magnetic bottle spectrometer, which inherently would preclude angular streaking. Likewise, increasing the angular sampling above about 6 would also deliver a diminishing return in reconstruction error; the 20 angular samples of the MRCOFFEE instrument are therefore a sufficient compromise for our goal of multiple energy windows. A similar approach to attoclock ptychography has been dubbed attosecond transient absorption 'spooktroscopy' for the sake of its relationship to classical ghost-imaging. Again, drawing inspiration from spatial domain methods for super-resolution, one uses high-resolution ultrafast absorption spectroscopy with broad-bandwidth XFEL pulses (Driver et al., 2020; Li et al., 2021) to recover the desired resolution in the time and energy domain. Other potential measurements involve the direct time-domain measurement of electronic relaxation dynamics in atoms and molecules as recently demonstrated for the Auger-Meitner electron decay dynamics in neon atoms (Haynes et al., 2021). One of our particular interests lies in temporally resolved measurements of resonant double-core hole processes and decay in both atomic and molecular species with respect to the X-ray pulse intensity substructure at the attosecond scale as statically initiated in recent work (Mazza et $a l ., 2020)$. Given the advances in optical pulse shaping for gasphase molecular alignment (Cryan et al., 2009) and orientation (Ren et al., 2014), we expect broad application of the method of using the rotational coherences induced by optically kicking a molecular rotor for extracting molecular-frame emission patterns from laboratory-frame fluctuations in yield (Marceau et al., 2017; Gregory et al., 2020). We therefore expect that the time-energy-resolved photoelectron and Auger-Meitner electron spectroscopy, reconstructed in the molecular frame, as enabled by our instrument, will find broad application for the investigation of nonlinear light-matter interactions and coherence effects at X-ray frequencies.

The molecular-frame angular-scattering pattern of ionized electrons, freed from the self same molecule, provides numerous benefits to conventional electron scattering (Boll et al., 2014; Nakajima et al., 2015; Kastirke et al., 2020). Recent investigations into the attosecond time-resolved photoionization process in molecules has shown that nuclear motion can in fact imprint its effect on the outgoing photoelectron. Such effects are only visible with sufficient time-energy resolution (Kamalov et al., 2020), ideally with reconstruction of the molecular-frame emission pattern since the topic of nuclear motion effects on the emission directions are a hot topic related to the extent of the validity of the Franck-Condon principle of molecular transitions (Nandi et al., 2020). Such recent attosecond photoionization measurements of mole- 
cular targets have underlined that molecular ionization is a complex process full of channel-coupling dynamics and rich physics as photoelectrons interact with the molecular orbitals as they re-arrange into cationic configurations. Studying the results of these fundamental scattering processes advances the understanding of electron dynamics in their natural time regime. Simulations of molecular-frame photoionization show complex angular dependencies that are difficult to measure with conventional detectors due to either a lack of angular resolution or poor energy resolution. The interpretation of existing measurements (Cryan et al., 2012) is also complicated due to partial-wave mixing that results from the stricter symmetry of molecules. The angular resolution of MRCOFFEE is sufficiently high to measure the laboratoryframe outgoing partial waves as they fluctuate with the coherent rotational wavepacket. The molecular-frame emission pattern is then reconstructed as per Gregory et al. (2020). Preserving high energy resolution makes this instrument ideal for molecular-frame photoionization measurements, enabling partial-wave decomposition of photo- and Auger-Meitner electron wavepackets. The high energy resolution over a wide energy window for each individual eToF detector is critical to mitigating the spectral breadth and congestion of the involved molecular cationic states. Another of the unique opportunities that XFELs offer is the possibility to site-specifically address individual constituents of a polyatomic system with ultraintense and ultrashort pulses, thereby enabling an observerspecific vista on nonlinear and ultrafast processes. A broad variety of new scientific routes has been initiated, and, in light of rapidly evolving technological and diagnostic advances on both the machine side and the experiment side, rich potential unfolds for the immediate and also longer-term future (Young et al., 2018; Ueda et al., 2019). A recent technological advance is undulator-based polarization control that enables efficient access to nonlinear and ultrafast dichroic phenomena in the X-ray regime (Allaria et al., 2014; Lutman et al., 2016b). The angular distribution of electrons emitted via circularly polarized light yields a variety of information on the ionizing light as well as access to scientific questions such as dichroic lightmatter interaction. The circularly polarizing 'Delta' afterburner undulator was successfully operated at LCLS I and has revealed promising scientific potential for using polarization control for ultrashort XFEL pulses (Lutman et al., 2016a; Hartmann et al., 2016). New schemes were recently proposed for enabling coherent X-rays with tunable time-dependent polarization (Sudar et al., 2020). Following the success of FERMI in Italy as the first polarization-controlling shortwavelength FEL, LCLS has pioneered polarization control at XFELs and enabled fundamental circular dichroism studies (Hartmann et al., 2016), magnetization studies via X-ray magnetic circular dichroism (XMCD) (Higley et al., 2016), as well as the first steps into studying dynamics and nonlinear processes in chiral systems with ultrabright X-ray pulses (Schoenlein et al., 2015; Ilchen et al., 2020). Several recent studies from FERMI give an appealing outlook on polarization control as extended to the X-ray regime (Mazza et al., 2014; Ilchen et al., 2017; Young et al., 2018; Ueda et al., 2019;
De Ninno et al., 2020; Carpeggiani et al., 2019). The upcoming opportunities include dichroism studies in the nonlinear regime (Ilchen et al., 2017; De Ninno et al., 2020) for new phenomena of elliptical dichroism (Hofbrucker et al., 2018) to non-dipole studies (Ilchen et al., 2018; Bachau \& Dieng, 2019; De Ninno et al., 2020), and the exploration of chirality via photoelectron circular dichroism (Ilchen et al., 2020; Ritchie, 1976; Wollenhaupt, 2016; Beaulieu et al., 2016). The unique advantage of the full MRCOFFEE spectrometer array is the potential to retrieve $3 \mathrm{D}$ angular-distribution patterns of mixed polarization states while maintaining the high energy resolution afforded by fast anode-mounted MCPs. The combination of undulator-based polarization control with intense attosecond pulses from XLEAP (Duris et al., 2020) can even add valuable perspectives for exploring electron dynamics in chiral systems before and right at the very onset of structural dynamics. This novel view on nonlinear and dynamical stereochemistry will strongly require exquisite knowledge of all underlying pulse properties such as the degree of polarization and the single-shot attosecond time-energy structure which underlines the core strengths of the instrument presented here.

\section{Conclusion and outlook}

We have presented the current layout and development of a new diagnostic and scientific instrument for atomic, molecular and optical physics at the TMO endstation of LCLS II. Some key advances compared with previous instruments targeting angle-resolving electron time-of-flight spectroscopy will enable and enhance non-invasive and online information about polarization control, spectral composition, and the attosecond time-energy structure of each incoming X-ray pulse. One of the core strengths of this novel spectrometer array is an optimized multi-resolution capability that allows for simultaneous investigation, within a single XFEL shot, of multiple different kinetic energy regimes. This instrument has been also designed for specific compatibility with $\mathrm{MHz}$ repetition rates, providing the development and demonstration platform for the upcoming technological advancements in widely controllable attosecond and novel structured X-ray pulses associated with the active pulse-shaping research for the LCLS II.

\section{Acknowledgements}

The authors want to thank Timor Osipov, Jeff Aldrich, Justin James, Amore Lopes, Paul Fuoss, Su-Ping Cheng, Alan Conder, James M. Glownia, Michael Holmes, David Rich, David Fritz, Gregor Hartmann, Markus Braune, Frank Scholz, and Jens Viefhaus, for their constant support and fruitful discussions. We thank Richard Davies (MDC Vacuum) for critical input into the design of the mu-metal chamber. We furthermore acknowledge the entire LCLS, LCLS II, L2SI subsystems and SLAC vacuum and machine shops staff for their assistance during the design, construction, and commissioning of the MRCOFFEE instrument. 


\section{Funding information}

This development and research was carried out at the Linac Coherent Light Source (LCLS) at the SLAC National Accelerator Laboratory. SLAC National Accelerator Laboratory, is supported by the US Department of Energy, Office of Science, Office of Basic Energy Sciences under Contract No. DE-AC02-76SF00515. RNC acknowledges funding through the US Department of Energy, Office of Science, Office of Basic Energy Sciences under Field Work Proposal 100498, 'Enabling long wavelength Streaking for Attosecond X-ray Science' as well as algorithm development support under Field Work Proposal 100643 'Actionable Information from Sensor to Data Center'. MI acknowledges funding of the Volkswagen foundation for a Peter-Paul-Ewald Fellowship.

\section{References}

Allaria, E., Diviacco, B., Callegari, C., Finetti, P., Mahieu, B., Viefhaus, J., Zangrando, M., De Ninno, G., Lambert, G., Ferrari, E., Buck, J., Ilchen, M., Vodungbo, B., Mahne, N., Svetina, C., Spezzani, C., Di Mitri, S., Penco, G., Trovó, M., Fawley, W. M., Rebernik, P. R., Gauthier, D., Grazioli, C., Coreno, M., Ressel, B., Kivimäki, A., Mazza, T., Glaser, L., Scholz, F., Seltmann, J., Gessler, P., Grünert, J., De Fanis, A., Meyer, M., Knie, A., Moeller, S. P., Raimondi, L., Capotondi, F., Pedersoli, E., Plekan, O., Danailov, M. B., Demidovich, A., Nikolov, I., Abrami, A., Gautier, J., Lüning, J., Zeitoun, P. \& Giannessi, L. (2014). Phys. Rev. X, 4, 041040.

Amann, J. (2018). Proceedings of the 38th International Free-Electron Laser Conference (FEL2017), 20-25 August 2017, Santa Fe, NM, USA.

Bachau, H. \& Dieng, M. (2019). Eur. Phys. J. D, 73, 123.

Beaulieu, S., Ferré, A., Géneaux, R., Canonge, R., Descamps, D., Fabre, B., Fedorov, N., Légaré, F., Petit, S., Ruchon, T., Blanchet, V., Mairesse, Y. \& Pons, B. (2016). New J. Phys. 18, 102002.

Becker, U., Szostak, D., Kerkhoff, H. G., Kupsch, M., Langer, B., Wehlitz, R., Yagishita, A. \& Hayaishi, T. (1989). Phys. Rev. A, 39, 3902-3911.

Berrah, N., Langer, B., Wills, A. A., Kukk, E., Bozek, J. D., Farhat, A. \& Gorczyca, T. W. (1999). J. Electron Spectrosc. Relat. Phenom. 101-103, 1-11.

Bionta, M. R., Hartmann, N., Weaver, M., French, D., Nicholson, D. J., Cryan, J. P., Glownia, J. M., Baker, K., Bostedt, C., Chollet, M., Ding, Y., Fritz, D. M., Fry, A. R., Kane, D. J., Krzywinski, J., Lemke, H. T., Messerschmidt, M., Schorb, S., Zhu, D., White, W. E. \& Coffee, R. N. (2014). Rev. Sci. Instrum. 85, 083116.

Boll, R., Rouzée, A., Adolph, M., Anielski, D., Aquila, A., Bari, S., Bomme, C., Bostedt, C., Bozek, J. D., Chapman, H. N., Christensen, L., Coffee, R., Coppola, N., De, S., Decleva, P., Epp, S. W., Erk, B., Filsinger, F., Foucar, L., Gorkhover, T., Gumprecht, L., Hömke, A., Holmegaard, L., Johnsson, P., Kienitz, J. S., Kierspel, T., Krasniqi, F., Kühnel, K., Maurer, J., Messerschmidt, M., Moshammer, R., Müller, N. L. M., Rudek, B., Savelyev, E., Schlichting, I., Schmidt, C., Scholz, F., Schorb, S., Schulz, J., Seltmann, J., Stener, M., Stern, S., Techert, S., Thøgersen, J., Trippel, S., Viefhaus, J., Vrakking, M., Stapelfeldt, H., Küpper, J., Ullrich, J., Rudenko, A. \& Rolles, D. (2014). Faraday Discuss. 171, 57-80.

Braune, M., Buck, J., Kuhlmann, M., Grunewald, S., Düsterer, S., Viefhaus, J. \& Tiedtke, K. (2018). J. Synchrotron Rad. 25, 3-15.

Braune, M., Hartmann, G., Ilchen, M., Knie, A., Lischke, T., Reinköster, A., Meissner, A., Deinert, S., Glaser, L., Al-Dossary, O., Ehresmann, A., Kheifets, A. S. \& Viefhaus, J. (2016). J. Mod. Opt. 63, 324-333.

Carpeggiani, P. A., Gryzlova, E. V., Reduzzi, M., Dubrouil, A., Faccialá, D., Negro, M., Ueda, K., Burkov, S. M., Frassetto, F.,
Stienkemeier, F., Ovcharenko, Y., Meyer, M., Plekan, O., Finetti, P., Prince, K. C., Callegari, C., Grum-Grzhimailo, A. N. \& Sansone, G. (2019). Nat. Phys. 15, 170-177.

Constant, E., Taranukhin, V., Stolow, A. \& Corkum, P. (1997). Phys. Rev. A, 56, 3870-3878.

Cryan, J. P., Bucksbaum, P. H. \& Coffee, R. N. (2009). Phys. Rev. A, 80, 063412.

Cryan, J. P., Glownia, J. M., Andreasson, J., Belkacem, A., Berrah, N., Blaga, C. I., Bostedt, C., Bozek, J., Buth, C., DiMauro, L. F., Fang, L., Gessner, O., Guehr, M., Hajdu, J., Hertlein, M. P., Hoener, M., Kornilov, O., Marangos, J. P., March, A. M., McFarland, B. K., Merdji, H., Petrović, V. S., Raman, C., Ray, D., Reis, D., Tarantelli, F., Trigo, M., White, J. L., White, W., Young, L., Bucksbaum, P. H. \& Coffee, R. N. (2010). Phys. Rev. Lett. 105, 083004.

Cryan, J. P., Glownia, J. M., Andreasson, J., Belkacem, A., Berrah, N., Blaga, C. I., Bostedt, C., Bozek, J., Cherepkov, N. A., DiMauro, L. F., Fang, L., Gessner, O., Gühr, M., Hajdu, J., Hertlein, M. P., Hoener, M., Kornilov, O., Marangos, J. P., March, A. M., McFarland, B. K., Merdji, H., Messerschmidt, M., Petrović, V. S., Raman, C., Ray, D., Reis, D. A., Semenov, S. K., Trigo, M., White, J. L., White, W., Young, L., Bucksbaum, P. H. \& Coffee, R. N. (2012). J. Phys. B At. Mol. Opt. Phys. 45, 055601.

De Ninno, G., Wätzel, J., Ribič, P. R., Allaria, E., Coreno, M., Danailov, M. B., David, C., Demidovich, A., Di Fraia, M., Giannessi, L., Hansen, K., Krušič, Š., Manfredda, M., Meyer, M., Mihelič, A., Mirian, N., Plekan, O., Ressel, B., Rösner, B., Simoncig, A., Spampinati, S., Stupar, M., Žitnik, M., Zangrando, M., Callegari, C. \& Berakdar, J. (2020). Nat. Photonics, 14, 554-558.

Driver, T., Li, S., Champenois, E. G., Duris, J., Ratner, D., Lane, T. J., Rosenberger, P., Al-Haddad, A., Averbukh, V., Barnard, T., Berrah, N., Bostedt, C., Bucksbaum, P. H., Coffee, R., DiMauro, L. F., Fang, L., Garratt, D., Gatton, A., Guo, Z., Hartmann, G., Haxton, D., Helml, W., Huang, Z., LaForge, A., Kamalov, A., Kling, M. F., Knurr, J., Lin, M. F., Lutman, A. A., MacArthur, J. P., Marangos, J. P., Nantel, M., Natan, A., Obaid, R., O'Neal, J. T., Shivaram, N. H., Schori, A., Walter, P., Li Wang, A., Wolf, T. J. A., Marinelli, A. \& Cryan, J. P. (2020). Phys. Chem. Chem. Phys. 22, 2704-2712.

Duris, J., Li, S., Driver, T., Champenois, E. G., MacArthur, J. P., Lutman, A. A., Zhang, Z., Rosenberger, P., Aldrich, J. W., Coffee, R., Coslovich, G., Decker, F. J., Glownia, J. M., Hartmann, G., Helml, W., Kamalov, A., Knurr, J., Krzywinski, J., Lin, M. F., Marangos, J. P., Nantel, M., Natan, A., O'Neal, J. T., Shivaram, N., Walter, P., Wang, A. L., Welch, J. J., Wolf, T. J., Xu, J. Z., Kling, M. F., Bucksbaum, P. H., Zholents, A., Huang, Z., Cryan, J. P. \& Marinelli, A. (2020). Nat. Photonics, 14, 30-36.

Eckle, P., Smolarski, M., Schlup, P., Biegert, J., Staudte, A., Schöffler, M., Muller, H. G., Dörner, R. \& Keller, U. (2008). Nat. Phys. 4, 565570.

Emma, C., Feng, Y., Nguyen, D. C., Ratti, A. \& Pellegrini, C. (2017). Phys. Rev. Accel. Beams, 20, 030701.

Goldsztejn, G., Marchenko, T., Céolin, D., Journel, L., Guillemin, R., Rueff, J.-P., Kushawaha, R. K., Püttner, R., Piancastelli, M. N. \& Simon, M. (2016). Phys. Chem. Chem. Phys. 18, 15133-15142.

Gregory, M., Hockett, P., Stolow, A. \& Makhija, V. (2020). arXiv:2012.04561 [physics. Chem-ph].

Hartmann, G., Lindahl, A. O., Knie, A., Hartmann, N., Lutman, A. A., MacArthur, J. P., Shevchuk, I., Buck, J., Galler, A., Glownia, J. M., Helml, W., Huang, Z., Kabachnik, N. M., Kazansky, A. K., Liu, J., Marinelli, A., Mazza, T., Nuhn, H.-D., Walter, P., Viefhaus, J., Meyer, M., Moeller, S., Coffee, R. N. \& Ilchen, M. (2016). Rev. Sci. Instrum. 87, 083113.

Hartmann, N., Hartmann, G., Heider, R., Wagner, M. S., Ilchen, M., Buck, J., Lindahl, A. O., Benko, C., Grünert, J., Krzywinski, J., Liu, J., Lutman, A. A., Marinelli, A., Maxwell, T., Miahnahri, A. A., Moeller, S. P., Planas, M., Robinson, J., Kazansky, A. K., Kabachnik, N. M., Viefhaus, J., Feurer, T., Kienberger, R., Coffee, R. N. \& Helml, W. (2018). Nat. Photon. 12, 215-220. 
Haynes, D. C., Wurzer, M., Schletter, A., Al-Haddad, A., Blaga, C., Bostedt, C., Bozek, J., Bromberger, H., Bucher, M., Camper, A., Carron, S., Coffee, R., Costello, J. T., DiMauro, L. F., Ding, Y., Ferguson, K., Grguraš, I., Helml, W., Hoffmann, M. C., Ilchen, M., Jalas, S., Kabachnik, N. M., Kazansky, A. K., Kienberger, R., Maier, A. R., Maxwell, T., Mazza, T., Meyer, M., Park, H., Robinson, J., Roedig, C., Schlarb, H., Singla, R., Tellkamp, F., Walker, P. A., Zhang, K., Doumy, G., Behrens, C. \& Cavalieri, A. L. (2021). Nat. Phys. 17, 512-518.

Heider, R., Wagner, M. S., Hartmann, N., Ilchen, M., Buck, J., Hartmann, G., Shirvanyan, V., Lindahl, A. O., Grünert, J., Krzywinski, J., Liu, J., Ossiander, M., Lutman, A. A., Maxwell, T., Miahnahri, A. A., Moeller, S. P., Planas, M., Robinson, J., Viefhaus, J., Feurer, T., Kienberger, R., Coffee, R. N. \& Helml, W. (2019). Phys. Rev. A, 100, 053420 .

Helml, W., Maier, A. R., Schweinberger, W., Grguraš, I., Radcliffe, P., Doumy, G., Roedig, C., Gagnon, J., Messerschmidt, M., Schorb, S., Bostedt, C., Grüner, F., DiMauro, L. F., Cubaynes, D., Bozek, J. D., Tschentscher, Th., Costello, J. T., Meyer, M., Coffee, R., Düsterer, S., Cavalieri, A. L. \& Kienberger, R. (2014). Nat. Photon. 8, 950957.

Hemmers, O., Whitfield, S. B., Glans, P., Wang, H., Lindle, D. W., Wehlitz, R. \& Sellin, I. A. (1998). Rev. Sci. Instrum. 69, 3809-3817.

Higley, D. J., Hirsch, K., Dakovski, G. L., Jal, E., Yuan, E., Liu, T., Lutman, A. A., MacArthur, J. P., Arenholz, E., Chen, Z., Coslovich, G., Denes, P., Granitzka, P. W., Hart, P., Hoffmann, M. C., Joseph, J., Le Guyader, L., Mitra, A., Moeller, S., Ohldag, H., Seaberg, M., Shafer, P., Stöhr, J., Tsukamoto, A., Nuhn, H. D., Reid, A. H., Dürr, H. A. \& Schlotter, W. F. (2016). Rev. Sci. Instrum. 87, 033110.

Hofbrucker, J., Volotka, A. V. \& Fritzsche, S. (2018). Phys. Rev. Lett. 121, 053401.

Hoppe, W. (1969). Acta Cryst. A25, 495-501.

Hoshino, M., Nakagawa, K., Makochekanwa, C., Tanaka, T., Kuze, N., Matsumoto, M., Fujiwara, K., De Fanis, A., Tamenori, Y., Kitajima, M., Tanaka, H. \& Ueda, K. (2006). Chem. Phys. Lett. 421, 256-260.

Ilchen, M., Douguet, N., Mazza, T., Rafipoor, A., Callegari, C., Finetti, P., Plekan, O., Prince, K., Demidovich, A., Grazioli, C., Avaldi, L., Bolognesi, P., Coreno, M., Di Fraia, M., Devetta, M., Ovcharenko, Y., Düsterer, S., Ueda, K., Bartschat, K., Grum-Grzhimailo, A., Bozhevolnov, A., Kazansky, A., Kabachnik, N. \& Meyer, M. (2017). Phys. Rev. Lett. 118, 013002.

Ilchen, M., Glaser, L., Scholz, F., Walter, P., Deinert, S., Rothkirch, A., Seltmann, J., Viefhaus, J., Decleva, P., Langer, B., Knie, A., Ehresmann, A., Al-Dossary, O. M., Braune, M., Hartmann, G., Meissner, A., Tribedi, L. C., AlKhaldi, M. \& Becker, U. (2014). Phys. Rev. Lett. 112, 023001.

Ilchen, M., Hartmann, G., Gryzlova, E. V., Achner, A., Allaria, E., Beckmann, A., Braune, M., Buck, J., Callegari, C., Coffee, R. N., Cucini, R., Danailov, M., De Fanis, A., Demidovich, A., Ferrari, E., Finetti, P., Glaser, L., Knie, A., Lindahl, A. O., Plekan, O., Mahne, N., Mazza, T., Raimondi, L., Roussel, E., Scholz, F., Seltmann, J., Shevchuk, I., Svetina, C., Walter, P., Zangrando, M., Viefhaus, J., Grum-Grzhimailo, A. N. \& Meyer, M. (2018). Nat. Commun. 9, 4659.

Ilchen, M., Schmidt, P., Novikovskiy, N. M., Hartmann, G., Rupprecht, P., Coffee, R. N., Ehresmann, A., Galler, A., Hartmann, N., Helml, W., Huang, Z., Inhester, L., Lutman, A. A., MacArthur, J. P., Maxwell, T., Meyer, M., Music, V., Nuhn, H.-D., Osipov, T., Ray, D., Wolf, T. J. A., Bari, S., Walter, P., Li, Z., Moeller, S., Knie, A. \& Demekhin, P. V. (2020). arXiv:2012.07100.

Kamalov, A., Wang, A. L., Bucksbaum, P. H., Haxton, D. J. \& Cryan, J. P. (2020). Phys. Rev. A, 102, 023118.

Kastirke, G., Schöffler, M. S., Weller, M., Rist, J., Boll, R., Anders, N., Baumann, T. M., Eckart, S., Erk, B., De Fanis, A., Fehre, K., Gatton, A., Grundmann, S., Grychtol, P., Hartung, A., Hofmann, M., Ilchen, M., Janke, C., Kircher, M., Kunitski, M., Li, X., Mazza, T., Melzer, N., Montano, J., Music, V., Nalin, G., Ovcharenko, Y., Pier, A., Rennhack, N., Rivas, D. E., Dörner, R., Rolles, D., Rudenko, A.,
Schmidt, P., Siebert, J., Strenger, N., Trabert, D., Vela-Perez, I., Wagner, R., Weber, T., Williams, J. B., Ziolkowski, P., Schmidt, L. P. H., Czasch, A., Trinter, F., Meyer, M., Ueda, K., Demekhin, P. V. \& Jahnke, T. (2020). Phys. Rev. X, 10, 021052.

Kazansky, A. K., Bozhevolnov, A. V., Sazhina, I. P. \& Kabachnik, N. M. (2016). Phys. Rev. A, 93, 013407.

Kienberger, R., Hentschel, M., Uiberacker, M., Spielmann, C., Kitzler, M., Scrinzi, A., Wieland, M., Westerwalbesloh, T., Kleineberg, U., Heinzmann, U., Drescher, M. \& Krausz, F. (2002). Science, 297, 1144-1148.

Kivimäki, A., Kukk, E., Karvonen, J., Mursu, J., Nõmmiste, E., Aksela, H. \& Aksela, S. (1998). Phys. Rev. A, 57, 2724-2730.

Laksman, J., Buck, J., Glaser, L., Planas, M., Dietrich, F., Liu, J., Maltezopoulos, T., Scholz, F., Seltmann, J., Hartmann, G., Ilchen, M., Freund, W., Kujala, N., Viefhaus, J. \& Grünert, J. (2019). J. Synchrotron Rad. 26, 1010-1016.

Li, S., Driver, T., Alexander, O., Cooper, B., Garratt, D., Marinelli, A., Cryan, J. P. \& Marangos, J. P. (2021). Faraday Discuss. 228, 488-501.

Li, S., Guo, Z., Coffee, R. N., Hegazy, K., Huang, Z., Natan, A., Osipov, T., Ray, D., Marinelli, A. \& Cryan, J. P. (2018). Opt. Express, 26, 4531-4547.

Lutman, A. A., Coffee, R., Ding, Y., Huang, Z., Krzywinski, J., Maxwell, T., Messerschmidt, M. \& Nuhn, H.-D. (2013). Phys. Rev. Lett. 110, 134801.

Lutman, A. A., MacArthur, J. P., Ilchen, M., Lindahl, A. O., Buck, J., Coffee, R. N., Dakovski, G. L., Dammann, L., Ding, Y., Dürr, H. A., Glaser, L., Grünert, J., Hartmann, G., Hartmann, N., Higley, D., Hirsch, K., Levashov, Y. I., Marinelli, A., Maxwell, T., Mitra, A., Moeller, S., Osipov, T., Peters, F., Planas, M., Shevchuk, I., Schlotter, W. F., Scholz, F., Seltmann, J., Viefhaus, J., Walter, P., Wolf, Z. R., Huang, Z. \& Nuhn, H. D. (2016a). Nat. Photon. 10, 468-472.

Lutman, A. A., Maxwell, T. J., MacArthur, J. P., Guetg, M. W., Berrah, N., Coffee, R. N., Ding, Y., Huang, Z., Marinelli, A., Moeller, S. \& Zemella, J. C. (2016b). Nat. Photon. 10, 745-750.

Marceau, C., Makhija, V., Platzer, D., Naumov, A. Y., Corkum, P. B., Stolow, A., Villeneuve, D. M. \& Hockett, P. (2017). Phys. Rev. Lett. 119, 083401.

Mazza, T., Ilchen, M., Kiselev, M., Gryzlova, E., Baumann, T., Boll, R., De Fanis, A., Grychtol, P., Montaño, J., Music, V., Ovcharenko, Y., Rennhack, N., Rivas, D., Schmidt, P., Wagner, R., Ziolkowski, P., Berrah, N., Erk, B., Johnsson, P., Küstner-Wetekam, C., Marder, L., Martins, M., Ott, C., Pathak, S., Pfeifer, T., Rolles, D., Zatsarinny, O., Grum-Grzhimailo, A. \& Meyer, M. (2020). Phys. Rev. X, 10, 041056.

Mazza, T., Ilchen, M., Rafipoor, A. J., Callegari, C., Finetti, P., Plekan, O., Prince, K. C., Richter, R., Danailov, M. B., Demidovich, A., De Ninno, G., Grazioli, C., Ivanov, R., Mahne, N., Raimondi, L., Svetina, C., Avaldi, L., Bolognesi, P., Coreno, M., O'Keeffe, P., Di Fraia, M., Devetta, M., Ovcharenko, Y., Möller, T., Lyamayev, V., Stienkemeier, F., Düsterer, S., Ueda, K., Costello, J. T., Kazansky, A. K., Kabachnik, N. M. \& Meyer, M. (2014). Nat. Commun. 5, 3648.

Nakajima, K., Teramoto, T., Akagi, H., Fujikawa, T., Majima, T., Minemoto, S., Ogawa, K., Sakai, H., Togashi, T., Tono, K., Tsuru, S., Wada, K., Yabashi, M. \& Yagishita, A. (2015). Sci. Rep. 5, 14065.

Nandi, S., Plésiat, E., Zhong, S., Palacios, A., Busto, D., Isinger, M., Neoričić, L., Arnold, C. L., Squibb, R. J., Feifel, R., Decleva, P., L'Huillier, A., Martín, F. \& Gisselbrecht, M. (2020). Sci. Adv. 6, eaba7762.

Nelder, J. A. \& Mead, R. (1965). Comput. J. 7, 308-313.

Patanen, M., Travnikova, O., Zahl, M. G., Söderström, J., Decleva, P., Thomas, T. D., Svensson, S., Mårtensson, N., Børve, K. J., Saethre, L. J. \& Miron, C. (2013). Phys. Rev. A, 87, 063420.

Ren, X., Makhija, V. \& Kumarappan, V. (2014). Phys. Rev. Lett. 112, 173602.

Ritchie, B. (1976). Phys. Rev. A, 14, 359-362.

Saalmann, U. \& Rost, J. M. (2020). Phys. Rev. Lett. 125, 113202. 
Schoenlein, R. et al. (2015). New Science Opportunities Enabled By LCLS II X-ray Lasers. Report SLAC-R-1053. SLAC National Accelerator Laboratory, Menlo Park, CA, USA.

Schweizer, T., Brügmann, M. H., Helml, W., Hartmann, N., Coffee, R. \& Feurer, T. (2018). Appl. Sci. 8, 1039.

Simon, M., Püttner, R., Marchenko, T., Guillemin, R., Kushawaha, R. K., Journel, L., Goldsztejn, G., Piancastelli, M. N., Ablett, J. M., Rueff, J.-P. \& Céolin, D. (2014). Nat. Commun. 5, 4069.

Sudar, N., Coffee, R. \& Hemsing, E. (2020). arXiv:2009.10914 [physics.Acc-ph].

Therrien, A. C., Herbst, R., Quijano, O., Gatton, A. \& Coffee, R. (2019). Proceedings of the 2019 IEEE Nuclear Science Symposium and Medical Imaging Conference, 26 October-2 November 2019, Manchester, UK, pp. 1-4.

Ueda, K., Sokell, E., Schippers, S., Aumayr, F., Sadeghpour, H., Burgdörfer, J., Lemell, C., Tong, X., Pfeifer, T., Calegari, F., Palacios, A., Martin, F., Corkum, P., Sansone, G., Gryzlova, E. V., Grum-Grzhimailo, A. N., Piancastelli, M. N., Weber, P. M., Steinle, T., Amini, K., Biegert, J., Berrah, N., Kukk, E., Santra, R., Müller,
A., Dowek, D., Lucchese, R. R., McCurdy, C. W., Bolognesi, P., Avaldi, L., Jahnke, T., Schöffler, M. S., Dörner, R., Mairesse, Y., Nahon, L., Smirnova, O., Schlathölter, T., Campbell, E. E. B., Rost, J., Meyer, M. \& Tanaka, K. A. (2019). J. Phys. B At. Mol. Opt. Phys. 52, 171001.

Viefhaus, J., Scholz, F., Deinert, S., Glaser, L., Ilchen, M., Seltmann, J., Walter, P. \& Siewert, F. (2013). Nucl. Instrum. Methods Phys. Res. A, 710, 151-154.

White, M. G., Rosenberg, R. A., Gabor, G., Poliakoff, E. D., Thornton, G., Southworth, S. H. \& Shirley, D. A. (1979). Rev. Sci. Instrum. 50, 1268-1273.

Wollenhaupt, M. (2016). New J. Phys. 18, 121001.

Young, L., Ueda, K., Gühr, M., Bucksbaum, P. H., Simon, M., Mukamel, S., Rohringer, N., Prince, K. C., Masciovecchio, C., Meyer, M., Rudenko, A., Rolles, D., Bostedt, C., Fuchs, M., Reis, D. A., Santra, R., Kapteyn, H., Murnane, M., Ibrahim, H., Légaré, F., Vrakking, M., Isinger, M., Kroon, D., Gisselbrecht, M., L'Huillier, A., Wörner, H. J. \& Leone, S. R. (2018). J. Phys. B At. Mol. Opt. Phys. 51, 032003. 